\section{Randall Rodríguez}

Costarricense. Licenciado en Psicología y Sociología de la Universidad de Costa Rica, UCR. Labora en el Instituto de Investigaciones Sociales de la Universidad de Costa Rica y está interesado en los temas de psicología social del desarrollo cognitivo, infancia y sociología de la educación.

\section{Adriana Salas}

Costarricense. Cursa la Maestría en Psicología de la Educación en la Universidad de Costa Rica. Licenciada en Psicología en la misma Universidad. Sus intereses investigativos están relacionados con el desarrollo humano, la infancia, los procesos de aprendizaje $y$ de pensamiento, la sexualidad, entre otros.

\section{Chester Urbina}

Costarricense. Actualmente docente en la Maestría en Derechos Humanos y Educación para la Paz, del Instituto de Estudios Latinoamericanos de la Universidad Nacional de Costa Rica.

\section{Marianela Román Solano}

Costarricense. Licenciada en Psicología de la Universidad de Costa Rica, actualmente trabaja para la Asociación Nacional de Educadores (ANDE), en promoción de la Salud Mental de docentes y participa en el área de investigación en dos proyectos relacionados con el ámbito educativo, uno referido a necesidades de formación permanente de educadores y el otro, atinente al bienestar psicológico docente en coordinación con el Instituto de Investigación en Educación de la Universidad de Costa Rica (INIE) y el Centro Nacional de Recursos para la Educación Inclusiva (CENAREC).

\section{Marjorie Moreno Salas}

Costarricense. Licenciada en Psicología, graduada de la Universidad de Costa Rica, actualmente cursa el Doctorado en Ciencias de la Educación de la Universidad Católica de Costa Rica. Ha laborado como investigadora para la Escuela de Psicología de la Universidad Católica de Costa Rica y el Instituto de Investigaciones Psicológicas de la UCR. Ha realizado diversas consultorías en el Área de Investigación y Juventud. Actualmente, desempeña labores de Docencia en la Escuela de Psicología de la Universidad de Costa Rica, y realiza actividades de Investigación y docencia para la Escuela de Psicología de la Universidad Católica de Costa Rica.

\section{Ángel Villafañe Santiago}

Puertorriqueño. Posee un doctorado en Educación con énfasis en Consejería de la 
Universidad Interamericana de Puerto Rico. Se desempeña como Consejero en Rehabilitación en el Departamento de Consejería para el Desarrollo Estudiantil de la Universidad de Puerto Rico, Recinto de Río Piedras. Ha trabajado como profesor a nivel graduado y como asesor en el área de personas con impedimentos. Áreas de interés en investigación: violencia, asistencia tecnológica para personas con impedimentos, selección vocacional y VIH/SIDA.

\section{María I. Jiménez Chafey}

Puertorriqueña. Posee un doctorado en Psicología Clínica de la Universidad Carlos Albizu, Recinto de San Juan en Puerto Rico. Se desempeña como psicóloga clínica en el Departamento de Consejería para el Desarrollo Estudiantil de la Universidad de Puerto Rico, Recinto de Río Piedras. Ha trabajado en las áreas de psicoterapia, evaluación, docencia, investigación y consultoría. Áreas de interés en investigación: tratamiento para la depresión en adolescentes, ideación y conducta suicida, psicología de la salud y violencia en las parejas.

\section{Manuel Capellas Casellas}

Puertorriqueño. Posee un doctorado en Educación con énfasis en Gerencia Educativa de la Universidad Interamericana de Puerto Rico. Actualmente se desempeña como profesor de matemáticas en la Universidad Politécnica de Puerto Rico y como evaluador externo de proyectos de innovación pedagógica en universidades tales como la Universidad Metropolitana del Sistema Universitario Ana G. Méndez. Sus áreas de estudio son múltiples y variadas, pero se enfoca principalmente en la evaluación de los procesos y productos educativos.

\section{Shirleen Collazo Santos}

Puertorriqueña. Doctorante en Psicología Clínica en la Universidad de Puerto Rico Recinto de Río Piedras. Bachiller en
Psicología y Salud Mental de la Universidad de Puerto Rico, Recinto de Ponce. Es asistente de Investigación en el Departamento de Consejería para el Desarrollo Estudiantil. Áreas de interés: el cuerpo en la clínica psicoanalítica y el trabajo del psicólogo clínico dentro del escenario hospitalario.

\section{Zaira Carvajal Orlich}

Costarricense. Licenciada en Psicología por la Universidad de Costa Rica. Máster en estudios de la Mujer por la Universidad de Costa Rica-Universidad Nacional. Es co-fundadora del Instituto de Estudios de la Mujer (IEM) de la Universidad Nacional. Es docente e investigadora del IEM sobre hostigamiento sexual, currículo oculto en educación y embarazo adolescente. Profesora invitada del ILANUD en el Programa contra la Violencia Doméstica, en Paraguay.

\section{Patricia Delvó Gutiérrez}

Costarricense. Licenciada en Estadística de la Universidad de Costa Rica. Bachiller y Máster en Administración de Empresas del Instituto Tecnológico de Costa Rica. Es profesora de la Universidad de Costa Rica e imparte cursos de estadística de nivel introductorio y medio para distintas carreras en dicha universidad. Ha participado en el IEM como investigadora en temas de hostigamiento sexual y violencia intrafamiliar.

\section{Susana Ruiz Seisdedos}

Española. Profesora Contratada. Doctora de la Universidad de Jaén. Doctora en Ciencia Política $y$ de la Administración. Licenciada en Derecho $y$ en Ciencias Políticas por la Universidad de Granada. Coordinadora del Máster en Gestión de la Cooperación Internacional y de las ONGDs. Subdirectora de Innovación y Calidad de la Escuela Universitaria de Trabajo Social de la Universidad de Jaén. 


\section{Petra Bonometti}

Italiana. Máster en Gestión de la Cooperación Internacional y de las ONGDs por la Universidad de Granada. Educadora social para la tutela de los menores de edad y la inclusión de grupos desfavorecidos. Licenciada en Ciencias Económicas y Empresariales por la Universidad de Brescia y en Ciencias de la Educación por la Universidad de Verona.

\section{Evelyn Castro Carmiol}

Costarricense. Máster en Estudios de Género y Cultura. Mención de Ciencias Sociales, Universidad de Chile. Licenciada en Trabajo Social de la Universidad de Costa Rica.

\section{Vanesa Vazquez Laba}

Argentina. Doctora en Ciencias Sociales de la Universidad de Buenos Aires. Magíster en Investigación en Ciencias Sociales (UBA). Lic. en Sociología (UBA). Docente de la Facultad de Ciencias Sociales de la Universidad de Buenos Aires (Argentina) y de la Universitat de les Illes Balears (España). Ha tenido distintas Becas de Investigación de la Universidad de Buenos Aires $y$ del Consejo Nacional de Investigaciones Científicas y Técnicas (CONICET). Se ha especializado en temas de trabajo femenino $y$ familia en el medio rural. Desde el año 2001 presenta trabajos en diferentes Congresos y Seminarios y tiene varias publicaciones.

\section{Teresita Cordero}

Costarricense. Doctora en Educación de la UCR. Es profesora catedrática, investigadora en el Instituto de Investigaciones en Educación (INIE) de la UCR y en el Centro de Investigación en Estudios de la Mujer. Su campo de estudios se vincula con la Psicología Social Comunitaria y los estudios culturales en el desarrollo infantil y el género. Actualmente es la directora del Instituto de Investigación en Educación (INIE).
Psicóloga de formación, con una Magíster Scientiae en Psicología (1996).

\section{Marlon Mora}

Costarricense. Doctor en Desigualdades e Intervención Social - graduación de honor Cum Laude en Universidad Pablo de Olavide, Sevilla, España-. Con posgrados en Interculturalidad y Diversidad, Derechos Humanos y Educación para la Paz. De formación básica comunicador. Académico de la Universidad Nacional y la Universidad de Costa Rica en diferentes programas de posgrado. Consultor de Naciones Unidas, ILANUD.

\section{Mario A. Ramírez}

Costarricense. Abogado. Licenciado en Derecho de la Universidad de Costa Rica. En la actualidad estudia en la Maestría Centroamericana de Sociología de la Facultad de Ciencias Sociales de dicha casa de estudios.

\section{Sofía Flores Solórzano}

Costarricense. Magister Scientiae en Comunicación, Sistema de Estudios de Posgrado, Universidad de Costa Rica. Licenciada en Lingüística, Facultad de Letras, Universidad de Costa Rica. Bachiller en Filología Hispánica, Facultad de Letras, Universidad de Costa Rica. Actualmente Docente de Literatura costarricense (Universidad Estatal a Distancia), de Humanidades (Universidad de Costa Rica) y coordinadora del Centro de Amigos para la Paz.

\section{Iván Molina}

Costarricense. Historiador. Catedrático de la Escuela de Historia. Investigador del Centro de Investigación en Identidad y Cultura Latinoamericanas (CIICLA), ambos de la Universidad de Costa Rica. Entre sus últimos libros, figuran: Los pasados de la memoria. El 
origen de la reforma social en Costa Rica (19381943) (Heredia, Editorial Universidad Nacional, 2008); y Ricardo Jiménez, (San José, Editorial Universidad Estatal a Distancia, 2009).

\section{Daniela Szuster}

Argentina. Licenciada en Psicología en la Universidad de Buenos Aires, Argentina. Máster en Ciencias Judaicas del Schechter Institute of Jewish Studies, Jerusalén (Israel) y finalizando Maestría en Estudios de la Mujer de la Universidad de Costa Rica y la Universidad Nacional de Costa Rica.

Ilustración de la portada: Jorge Solano Cantillo

Costarricense. Bachiller en Psicología de la Universidad de Costa Rica. Prácticas profesionales e investigaciones en Psicología y otras carreras desde el año 2005. Ilustrador de materiales gráficos e instrumentos de aplicación para trabajos finales de graduación. Profesor de Novela Gráfica-ComicManga en el centro Casa del Adolescente Saint-Exupery, Pavas. 